Discrete Comput Geom 34:1-10 (2005)

DOI: $10.1007 / \mathrm{s} 00454-004-1110-\mathrm{x}$

Geometry

\title{
Forbidden Families of Geometric Permutations in $\mathbb{R}^{d *}$
}

\author{
Andrei Asinowski and Meir Katchalski \\ Faculty of Mathematics, Technion - Israel Institute of Technology, \\ Haifa 32000, Israel \\ \{andrei,meirk\}@techunix.technion.ac.il
}

\begin{abstract}
A geometric permutation induced by a transversal line of a finite family $\mathcal{F}$ of disjoint convex sets in $\mathbb{R}^{d}$ is the order in which the transversal meets the members of the family. We prove that for each natural $k$, each family of $k$ permutations is realizable (as a family of geometric permutations of some $\mathcal{F}$ ) in $\mathbb{R}^{d}$ for $d \geq 2 k-1$, but there is a family of $k$ permutations which is non-realizable in $\mathbb{R}^{d}$ for $d \leq 2 k-2$.
\end{abstract}

\section{Introduction}

Let $\mathcal{F}=\left\{A_{1}, A_{2}, \ldots, A_{n}\right\}$ be a finite family of $n$ pairwise disjoint convex sets in $\mathbb{R}^{d}$. A line $l$ is a transversal of $\mathcal{F}$ if it intersects all the members of $\mathcal{F}$. Each non-directed transversal intersects the members of $\mathcal{F}$ in an order that can be described by a pair of permutations of $\{1,2, \ldots, n\}$ which are reverses of each other. Such a pair is called a geometric permutation. Figure 1 shows an example of a planar family of four sets that admits six geometric permutations.

Earlier results on geometric permutations deal mostly with a maximal number of geometric permutations that a family of $n$ disjoint convex sets can have. For example, in $\mathbb{R}^{2}$ this number is $2 n-2$ (for $n \geq 4$ ) [5], [10], and in $\mathbb{R}^{d}$ it is known to be $O\left(n^{2 d-2}\right.$ ) [15] and $\Omega\left(n^{d-1}\right)$ [9]. For $n$ disjoint balls in $\mathbb{R}^{d}$ the maximal number of geometric permutations is $\Theta\left(n^{d-1}\right)$ [13]; and if the balls are congruent then this maximal number is bounded by a constant [11] (improved in [3] to 2 for $n \geq 9$ ). With the restriction that the members of the family are translates of a convex set in $\mathbb{R}^{2}$, the maximal number of geometric permutations is 3 [8], [9], and a complete characterization of possible families of geometric permutations in this case is known [1]. In contrast, the maximal number of

* These results are part of Andrei Asinowski's Ph.D. Thesis written under the supervision of Meir Katchalski. 


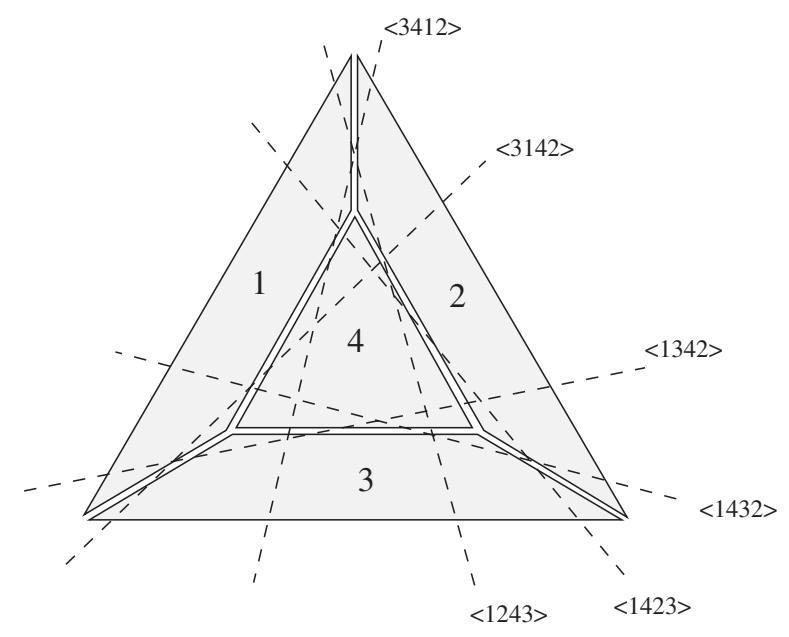

Fig. 1. A family of four sets in $\mathbb{R}^{2}$ with six geometric permutations.

geometric permutations for all families of $n$ disjoint translates of a convex set in $\mathbb{R}^{3}$ is $\Omega(n)[2]$.

One of the motivations for studying geometric permutations is Helly-type problems on the existence of common transversals for families of disjoint translates of a convex set: see, for example, Tverberg's solution of Grünbaum's conjecture about transversals of disjoint translates in $\mathbb{R}^{2}$ [14], and a Helly-type result for disjoint unit balls in $\mathbb{R}^{3}$ [7]. For other related results see [4], [6] and [16].

This paper deals with the following aspect of geometric permutations. Let $\mathcal{P}=$ $\left\{p_{1}, p_{2}, \ldots, p_{k}\right\}$ be some family of permutations on $n$ sets (throughout this paper we assume that all of the permutations are distinct, and, furthermore, no two of them are reverses of each other). It can be asked whether there is a family $\mathcal{F}$ that admits all of them as its geometric permutations. The answer may depend on $d$ (the dimension of the space). If there is such a family in $\mathbb{R}^{d}$, we say that $\mathcal{P}$ is realizable in $\mathbb{R}^{d}$, otherwise we say that it is non-realizable (or forbidden) in $\mathbb{R}^{d}$.

For example, the pair of permutations $\{\langle 1234\rangle,\langle 2143\rangle\}$ is forbidden in $\mathbb{R}^{2}$ (see Example 1 in Section 3.2). On the other hand, for each natural $n$, any pair $\left\{p_{1}, p_{2}\right\}$ of permutations on $n$ sets is realizable in $\mathbb{R}^{3}$ (this is a special case of Theorem 1).

Our results deal with realizable and forbidden families of permutations in $\mathbb{R}^{d}$ :

Theorem 1. For each natural $k$, each family of $k$ permutations is realizable in $\mathbb{R}^{2 k-1}$.

Theorem 2. For each natural $k$, there is a family of $k$ permutations which is nonrealizable in $\mathbb{R}^{2 k-2}$.

It is clear that if a family of permutations is realizable in $\mathbb{R}^{d}$, then it is realizable in each $\mathbb{R}^{d^{\prime}}$ where $d^{\prime} \geq d$. Thus it follows from our theorems that each family of $k$ permutations is realizable in $\mathbb{R}^{d}$ for each $d \geq 2 k-1$, but there is a family of $k$ permutations which is non-realizable in $\mathbb{R}^{d}$ for each $d \leq 2 k-2$. 


\section{Proof of Theorem 1}

Let $\left\{p_{1}, p_{2}, \ldots, p_{k}\right\}$ be a family of permutations on $n$ sets. Take $k$ lines $l_{1}, l_{2}, \ldots, l_{k}$ in general position in $\mathbb{R}^{2 k-1}$ (by general position we mean that their affine hull is $\mathbb{R}^{2 k-1}$ ). For each $j \in\{1,2, \ldots, k\}$, put $n$ points $P_{j 1}, P_{j 2}, \ldots, P_{j n}$ on $l_{j}$, according to the permutation $p_{j}$. For each $i \in\{1,2, \ldots, n\}$, define $S_{i}=\operatorname{conv}\left(\left\{P_{1 i}, P_{2 i}, \ldots, P_{k i}\right\}\right)$. Each $S_{i}$ is of dimension at most $k-1$.

We prove that the sets $S_{1}, S_{2}, \ldots, S_{n}$ are pairwise disjoint: suppose $S_{x} \cap S_{y} \neq \varnothing$ with $x \neq y$. Let $\tau$ be the minimal flat containing $S_{x}$ and $S_{y}$. Since $S_{x}$ and $S_{y}$ intersect, the dimension of $\tau$ is at most $2 k-2$. Then for each $j \in\{1,2, \ldots, k\}$, the points $P_{j x}$ and $P_{j y}$, and therefore the line $l_{j}$, belong to $\tau$. Thus all the lines $l_{1}, l_{2}, \ldots, l_{k}$ lie in $\tau$, contradicting their being in general position.

Thus $\left\{S_{1}, S_{2}, \ldots, S_{n}\right\}$ is a family of pairwise disjoint convex sets in $\mathbb{R}^{2 k-1}$, and it has $p_{1}, p_{2}, \ldots, p_{k}$ (induced by $l_{1}, l_{2}, \ldots, l_{k}$, respectively) as geometric permutations.

\section{Proof of Theorem 2}

\subsection{Notations}

Let $\left\{A_{1}, A_{2}, \ldots, A_{n}\right\}$ be a family of disjoint convex sets in $\mathbb{R}^{d}$, and let $l$ be a transversal of this family.

We write $l:\left(A_{x_{1}} \prec A_{x_{2}} \prec \cdots \prec A_{x_{n}}\right)$, or just $l:\left(x_{1} \prec x_{2} \prec \cdots \prec x_{n}\right)$, if $l$ is directed, and it meets these sets in this order: $A_{x_{1}}$ before $A_{x_{2}}$ before $\cdots$ before $A_{x_{n}}$.

We write $l:\left(A_{x_{1}} * A_{x_{2}} * \cdots * A_{x_{n}}\right)$, or just $l:\left(x_{1} * x_{2} * \cdots * x_{n}\right)$, either if $l$ is directed and $l:\left(A_{x_{1}} \prec A_{x_{2}} \prec \cdots \prec A_{x_{n}}\right)$ or $l:\left(A_{x_{n}} \prec A_{x_{n-1}} \prec \cdots \prec A_{x_{1}}\right)$, or if $l$ is undirected but this happens when we choose a direction on it. Of course, $l$ : $\left(A_{x_{1}} * A_{x_{2}} * \cdots * A_{x_{n}}\right)$ is the same as $l:\left(A_{x_{n}} * \cdots * A_{x_{2}} * A_{x_{1}}\right)$, and $l:\left(A_{x} * A_{y} * A_{z}\right)$ means simply that on $l$, $l \cap A_{y}$ is between $l \cap A_{x}$ and $l \cap A_{z}$.

For two disjoint convex sets $A_{x}$ and $A_{y}$ in $\mathbb{R}^{d}$, we denote by $H^{(x y)}$ a hyperplane $((d-$ 1)-flat) that strictly separates $A_{x}$ from $A_{y}$; by $H_{x}^{(x y)}$ the open halfspace bounded by $H^{(x y)}$ that contains $A_{x}$, and by $H_{y}^{(x y)}$ the open halfspace bounded by $H^{(x y)}$ that contains $A_{y}$.

\subsection{Examples of Forbidden Families of Permutations in $\mathbb{R}^{2}, \mathbb{R}^{3}$ and $\mathbb{R}^{4}$}

In this section we provide three examples of forbidden families of permutations. Examples 1 and 3 illustrate the general idea used in the proof of Theorem 2; Example 2 illustrates a slightly different method, applied to $\mathbb{R}^{3}$.

Example 1. The pair of permutations

$$
\begin{aligned}
\left\{p_{1}\right. & =\left\langle\begin{array}{ll}
12 & 34
\end{array},\right. \\
p_{2} & =\left\langle\begin{array}{ll}
21 & 43
\end{array}\right\}
\end{aligned}
$$

is forbidden in $\mathbb{R}^{2}$. 
Remark. This example already appeared in early papers on geometric permutations [8], [10].

Proof. Suppose that this pair is realizable in $\mathbb{R}^{2}$ with $\mathcal{F}=\left\{A_{1}, A_{2}, A_{3}, A_{4}\right\}$ and transversal lines $l_{1}, l_{2}$ inducing permutations $p_{1}, p_{2}$ respectively. Since parallel transversals clearly induce the same permutation, $l_{1}$ and $l_{2}$ intersect in a point $O$. Note that for each possible position of $O$ on the transversals relative to the members of $\mathcal{F}$, there exist $A_{x}$ and $A_{y}$ in $\mathcal{F}$ so that $l_{1}:(O * x * y)$ and $l_{2}:(O * y * x)$, and this contradicts the disjointness of the sets (the bar $\mid$ denotes the position of $O$ on the transversals; * is dropped):

- $l_{1}:(|1| 2 \mid 34), l_{2}:(|2| 1 \mid 43):$ take $x=3, y=4$.

- $l_{1}:(12|3| 4 \mid), l_{2}:(21|4| 3 \mid):$ take $x=2, y=1$.

- $l_{1}:(|1| 234), l_{2}:(214|3|)$ : take $x=2, y=4$.

- $l_{1}:(123|4|), l_{2}:(|2| 143)$ : take $x=3, y=1$.

Example 2. The triple of permutations

$$
\begin{aligned}
& \left\{p_{1}=\left\langle\begin{array}{ll}
123 & 456
\end{array},\right.\right. \\
& p_{2}=\left\langle\begin{array}{lll}
321 & 654
\end{array}\right\rangle \text {, } \\
& p_{3}=\left\langle\begin{array}{lll}
246 & 135
\end{array}\right\}
\end{aligned}
$$

is forbidden in $\mathbb{R}^{3}$.

Proof. Suppose that this triple is realizable in $\mathbb{R}^{3}$ with $\mathcal{F}=\left\{A_{1}, A_{2}, \ldots, A_{6}\right\}$ and transversal lines $l_{1}, l_{2}, l_{3}$ inducing permutations $p_{1}, p_{2}, p_{3}$, respectively. Using standard arguments (we mention them later in Section 3.4), it is possible to assume that no two lines among $l_{1}, l_{2}$ and $l_{3}$ intersect, and that there is no plane parallel to all of them. Then there exists a line $m$ which is parallel to $l_{3}$ and intersects both $l_{1}$ and $l_{2}$-in points $O_{1}$ and $O_{2}$, respectively (such a line $m$ exists since the plane $\tau$ that contains $l_{1}$ and is parallel to $l_{3}$, intersects $l_{2}$ in a point; denote this point by $O_{2} ; m$ is the line parallel to $l_{3}$ that contains $\left.O_{2}\right)$. Choose a direction for $m$, and the same direction for $l_{3}$, so that $m:\left(O_{1} \prec O_{2}\right)$.

Suppose that there exist $A_{x}, A_{y} \in \mathcal{F}$ so that $l_{1}:\left(O_{1} * A_{x} * A_{y}\right)$ and $l_{2}:\left(O_{2} * A_{y} * A_{x}\right)$. This implies $O_{1} \in H_{x}^{(x y)}$ and $O_{2} \in H_{y}^{(x y)}$, hence $m:\left(H_{x}^{(x y)} \prec H_{y}^{(x y)}\right)$, and thus also $l_{3}:\left(H_{x}^{(x y)} \prec H_{y}^{(x y)}\right)$. However, $l_{3}$ is a transversal of $\mathcal{F}$, hence $l_{3}:\left(A_{x} \prec A_{y}\right)$.

Note that for each possible position of $O_{1}$ and $O_{2}$ on the transversals relative to the members of $\mathcal{F}$, we can choose two pairs of members of $\mathcal{F}$ so that the previous observation contradicts the actual permutation $p_{3}$ (the bar | denotes the position of $O_{j}$ on the transversal $l_{j}$ ):

- $l_{1}:(|1| 23456)$ and $l_{2}:(|4| 5 \mid 6123)$.

$l_{1}$ : (|26) and $l_{2}$ : (|62) imply $l_{3}:(2 \prec 6) ; l_{1}$ : (|36) and $l_{2}$ : (|63) imply $l_{3}$ : $(3 \prec 6)$.

A contradiction to $l_{3}:(2 * 6 * 3)$.

- $l_{1}:(12 \mid 3456)$ and $l_{2}:(|4| 5 \mid 6123)$.

$l_{1}$ : (|21) and $l_{2}$ : (|12) imply $l_{3}:(2 \prec 1) ; l_{1}$ : (|36) and $l_{2}$ : (|63) imply $l_{3}$ : $(3 \prec 6)$.

A contradiction to $l_{3}:(2 * 6 * 1 * 3)$. 
- $l_{1}:(|1| 2|3| 456)$ and $l_{2}:(456|1| 2|3|)$.

$l_{1}$ : (|46) and $l_{2}$ : (|64) imply $l_{3}:(4 \prec 6) ; l_{1}:(\mid 56)$ and $l_{2}:(\mid 65)$ imply $l_{3}:(5 \prec 6)$.

A contradiction to $l_{3}:(4 * 6 * 5)$.

- $l_{1}:(123|4| 5|6|)$ and $l_{2}:(|4| 5|6| 123)$.

$l_{1}:(\mid 21)$ and $l_{2}$ : (|12) imply $l_{3}:(2 \prec 1) ; l_{1}:(\mid 31)$ and $l_{2}$ :(|13) imply $l_{3}:(3 \prec 1)$. A contradiction to $l_{3}:(2 * 1 * 3)$.

- $l_{1}:(1234 \mid 56)$ and $l_{2}:(4561|2| 3 \mid)$.

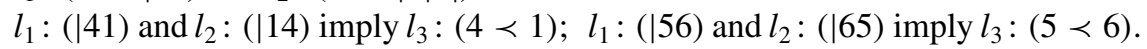
A contradiction to $l_{3}:(4 * 6 * 1 * 5)$.

- $l_{1}:(12345|6|)$ and $l_{2}:(4561|2| 3 \mid)$.

$l_{1}$ : (|41) and $l_{2}:(\mid 14)$ imply $l_{3}:(4 \prec 1) ; l_{1}:(\mid 51)$ and $l_{2}:(\mid 15)$ imply $l_{3}:(5 \prec 1)$. A contradiction to $l_{3}:(4 * 1 * 5)$.

Remark. In this forbidden triple, replacing $p_{3}$ by one of the seven permutations obtained from it by rearranging some of the pairs $\{2,4\},\{1,6\},\{3,5\}$ (for example $\langle 421635\rangle$ ) also gives a forbidden triple. This can be proved using the same method.

Example 3. The triple of permutations

$$
\begin{aligned}
\left\{p_{1}\right. & =\left\langle\begin{array}{lll}
123 & 456 & 789
\end{array},\right. \\
p_{2} & =\left\langle\begin{array}{llll}
312 & 564 & 978
\end{array},\right. \\
p_{3} & =\left\langle\begin{array}{llll}
231 & 645 & 897
\end{array}\right\}
\end{aligned}
$$

is forbidden in $\mathbb{R}^{4}$.

Proof. Suppose that this triple is realizable in $\mathbb{R}^{4}$ with $\mathcal{F}=\left\{A_{1}, A_{2}, \ldots, A_{9}\right\}$ and transversal lines $l_{1}, l_{2}, l_{3}$ inducing permutations $p_{1}, p_{2}, p_{3}$, respectively. It is possible to assume that there is a line $s$ that intersects each of $l_{1}, l_{2}, l_{3}$ (this follows from Lemma 4 to be proved later). For $i \in\{1,2,3\}$, choose a point $O_{i} \in l_{i} \cap s$. It is also possible to assume that $O_{3} \in \operatorname{conv}\left(\left\{O_{1}, O_{2}\right\}\right)$ : it is easy to see, using the symmetry of the permutations, that the two other possibilities can be obtained from this by relabeling the sets. Note that for each possible position of $O_{1}, O_{2}, O_{3}$ on the transversals relative to the members of $\mathcal{F}$, there exist $A_{x}$ and $A_{y}$ in $\mathcal{F}$ so that $l_{1}:\left(O_{1} * x * y\right)$ and $l_{2}:\left(O_{2} * x * y\right)$, but $l_{3}:\left(O_{3} * y * x\right)$ :

- $l_{1}:(|1| 2|3| 4|5| 6 \mid 789), l_{2}:(|3| 1|2| 5|6| 4|9| 78), l_{3}:(|2| 3|1| 6|4| 5 \mid 897)$ : take $x=7$, $y=8$.

- $l_{1}:(12|3| 4|5| 6|7| 8|9|), l_{2}:(312|5| 6|4| 9|7| 8 \mid), l_{3}:(231|6| 4|5| 8|9| 7 \mid):$ take $x=2$, $y=1$.

- $l_{1}:(|1| 2|3| 4|5| 6789), l_{2}:(|3| 1|2| 5 \mid 64978), l_{3}:(2316458|9| 7 \mid)$ : take $x=6, y=8$.

- $l_{1}:(12345|6| 7|8| 9 \mid), l_{2}:(3125|6| 4|9| 7|8|), l_{3}:(|2| 3 \mid 1645897)$ : take $x=5, y=1$.

- $l_{1}:(|1| 2|3| 456789), l_{2}:(|3| 1|2| 5|6| 4978), l_{3}:(2316458|9| 7 \mid)$ : take $x=4, y=8$.

- $l_{1}:(1234|5| 6|7| 8|9|), l_{2}:(312564|9| 7|8|), l_{3}:(|2| 3 \mid 1645897)$ : take $x=4, y=1$.

- $l_{1}$ : (|1|2|3|456789), $l_{2}:(312564|9| 7|8|), l_{3}:(231645|8| 9|7|)$ : take $x=4, y=5$.

- $l_{1}:(|1| 2|3| 456789), l_{2}:(312564|9| 7|8|), l_{3}:(|2| 3|1| 645897)$ : take $x=4, y=6$.

- $l_{1}:(|1| 2|3| 4|5| 6 \mid 789), l_{2}:(31256497|8|), l_{3}:(|2| 3|1| 6|4| 5|8| 97)$ : take $x=7$, $y=9$. 
- $l_{1}:(|1| 23456789), l_{2}:(312|5| 6|4| 9|7| 8 \mid), l_{3}:(23|1| 6|4| 5|8| 9|7|):$ take $x=2$, $y=3$.

- $l_{1}:(123456|7| 8|9|), l_{2}:(|3| 1|2| 5 \mid 64978), l_{3}:(23164|5| 8|9| 7 \mid):$ take $x=6, y=4$.

- $l_{1}:(12345|6| 7|8| 9 \mid), l_{2}:(|3| 1|2| 564978), l_{3}:(|2| 3|1| 6 \mid 45897)$ : take $x=5, y=4$.

This contradicts the disjointness of the members of $\mathcal{F}: O_{1}, O_{2} \in H_{x}^{(x y)}$, and $O_{3} \in H_{y}^{(x y)}$. However, also $O_{3} \in H_{x}^{(x y)}$ since $O_{3} \in \operatorname{conv}\left(\left\{O_{1}, O_{2}\right\}\right)$. Thus, $O_{3}$ belongs both to $H_{x}^{(x y)}$ and to $H_{y}^{(x y)}$, a contradiction.

\subsection{A $(k-2)$-Flat that Intersects All the Transversals}

We prove two lemmas that imply the existence of a $(k-2)$-flat that intersects a transversal line for each of the $k$ geometric permutations.

Definition. A family $\mathcal{L}$ of $s$-flats in $\mathbb{R}^{d}$ is an open family if for any $L \in \mathcal{L}$, there are $s+1$ open balls $B_{1}, B_{2}, \ldots, B_{s+1}$ so that $L$ intersects each of them, and any $s$-flat that intersects all these balls belongs to $\mathcal{L}$.

Remark. This definition implies that for each member of an open family, a small perturbation results in another member of the family. For $s=0$, an open family (of points) is just an open set in the usual sense.

Lemma 3. Let $k \in \mathbb{N}$. Let $\mathcal{L}_{1}, \mathcal{L}_{2}, \ldots, \mathcal{L}_{k}$ be open families of lines in $\mathbb{R}^{2 k-1}$, and let $P$ be a point in $\mathbb{R}^{2 k-1}$. Then there exist lines $l_{i} \in \mathcal{L}_{i}$ and a $(k-1)$-flat $S$ so that $P \in S$ and for each $i \in\{1,2, \ldots, k\}, l_{i}$ intersects $S$.

Lemma 4. Let $k \in \mathbb{N}, k>1$. Let $\mathcal{L}_{1}, \mathcal{L}_{2}, \ldots, \mathcal{L}_{k}$ be open families of lines in $\mathbb{R}^{2 k-2}$. Then there exist lines $l_{i} \in \mathcal{L}_{i}$, and a $(k-2)$-flat $S$ so that for each $i \in\{1,2, \ldots, k\}, l_{i}$ intersects $S$.

Proof of Lemma 3. For $k=1$ the statement is obvious.

Suppose the lemma holds for $k-1$.

For $1 \leq i \leq k$, it is possible to choose $l_{i} \in \mathcal{L}_{i}$ so that: $A=\operatorname{aff}\left\{l_{1}, l_{2}, \ldots, l_{k-1}\right\}$ is a (2k-3)-flat; $B=\operatorname{aff}\left\{l_{k}, P\right\}$ is a 2-flat; and $A \cap B$ is a point $Q$ different from $P$, so that the line $P Q$ intersects $l_{k}$.

For $1 \leq i \leq k-1$, let $\mathcal{L}_{i}^{\prime}=\mathcal{L}_{i} \cap A$. Each $\mathcal{L}_{i}^{\prime}$ is an open family of lines relative to $A$. By the induction assumption applied to $\mathcal{L}_{1}^{\prime}, \mathcal{L}_{2}^{\prime}, \ldots, \mathcal{L}_{k-1}^{\prime}$ and $Q$ in the $(2 k-3)$-flat $A$, there exist $l_{i}^{\prime} \in \mathcal{L}_{i}^{\prime} \subseteq \mathcal{L}_{i}(1 \leq i \leq k-1)$, and a $(k-2)$-flat $T$ that contains $Q$ and intersects each $l_{i}^{\prime}$.

Let $S=\operatorname{aff}(T, P)$. Clearly, $P \in S$. The flat $S$ intersects each $l_{i}^{\prime}$ since $T \subseteq S$, and it intersects $l_{k}$ since the line $P Q$ lies in $S$. Since $Q$ is the only point in $A \cap B, S$ is a $(k-1)$-flat.

Thus, $S$ and the lines $l_{1}^{\prime}, l_{2}^{\prime}, \ldots, l_{k-1}^{\prime}, l_{k}$ satisfy the conclusion of the lemma. 
Proof of Lemma 4. For $1 \leq i \leq k$, it is possible to choose $l_{i} \in \mathcal{L}_{i}$ so that $C=$ $\operatorname{aff}\left\{l_{1}, l_{2}, \ldots, l_{k-1}\right\}$ is a $(2 k-3)$-flat, and $l_{k} \cap C$ is a point $P$.

For $1 \leq i \leq k-1$, let $\mathcal{L}_{i}^{\prime}=\mathcal{L}_{i} \cap C$. Each $\mathcal{L}_{i}^{\prime}$ is an open family of lines in $C$. By Lemma 3 applied to $\mathcal{L}_{1}^{\prime}, \mathcal{L}_{2}^{\prime}, \ldots, \mathcal{L}_{k-1}^{\prime}$ and $P$ in the $(2 k-3)$-flat $C$, there exist $l_{i}^{\prime} \in \mathcal{L}_{i}^{\prime}$ $(1 \leq i \leq k-1)$, and a $(k-2)$-flat $S$ that intersects each $l_{i}^{\prime}$ and contains $P$ (that belongs to $l_{k}$ ).

Thus, $S$ and the lines $l_{1}^{\prime}, l_{2}^{\prime}, \ldots, l_{k-1}^{\prime}, l_{k}$ satisfy the conclusion of the lemma.

\subsection{Idea of the Proof of Theorem 2}

Let $\mathcal{F}=\left\{A_{1}, A_{2}, \ldots, A_{n}\right\}$ be a family of disjoint convex sets in $\mathbb{R}^{2 k-2}$ that has permutations $p_{1}, p_{2}, \ldots, p_{k}$. After a slight expansion of the members of $\mathcal{F}$, for each geometric permutation there is a transversal line that intersects all the members of $\mathcal{F}$ in interior points. Then, for each $i$, the family of all the transversal lines that induce $p_{i}$ contains an open family of lines. Hence, by Lemma 4 , it is possible to choose transversals $l_{1}, l_{2}, \ldots, l_{k}$ (inducing $p_{1}, p_{2}, \ldots, p_{k}$, respectively) so that there is a $(k-2)$-dimensional flat $S$ that intersects each of these transversals.

For each $j \in\{1,2, \ldots, k\}$, let $O_{i} \in l_{i} \cap S$. These are $k$ points in a $(k-2)$-flat, thus by Radon's theorem [12] they can be partitioned into two non-empty sets whose convex hulls intersect: $\{1,2, \ldots, k\}=K \cup L, K \cap L=\emptyset, K, L \neq \emptyset$, and $\operatorname{conv}\left(\left\{O_{j}: j \in\right.\right.$ $K\}) \cap \operatorname{conv}\left(\left\{O_{j}: j \in L\right\}\right) \neq \varnothing$.

Suppose that there are two sets $A_{x}$ and $A_{y}$ in $\mathcal{F}$ so that for each $j \in K, l_{j}:\left(O_{j} * A_{x} *\right.$ $\left.A_{y}\right)$, and for each $j \in L, l_{j}:\left(O_{j} * A_{y} * A_{x}\right)$. Then for each $j \in K, O_{j} \in H_{x}^{(x y)}$, and for each $j \in L, O_{j} \in H_{y}^{(x y)}$. Since the open halfspaces $H_{x}^{(x y)}$ and $H_{y}^{(x y)}$ are convex sets, it follows that $\operatorname{conv}\left(\left\{O_{j}: j \in K\right\}\right) \subseteq H_{x}^{(x y)}$ and $\operatorname{conv}\left(\left\{O_{j}: j \in L\right\}\right) \subseteq H_{y}^{(x y)}$. However, then each point common to $\operatorname{conv}\left(\left\{O_{j}: j \in K\right\}\right)$ and $\operatorname{conv}\left(\left\{O_{j}: j \in L\right\}\right)$ belongs to both $H_{x}^{(x y)}$ and $H_{y}^{(x y)}$, which is clearly impossible.

Thus, we have proved the following:

Observation 5. If a family of permutations $\left\{p_{1}, p_{2}, \ldots, p_{k}\right\}$ for $\mathcal{F}$ is such that for each partition of $\{1,2, \ldots, k\}$ into two disjoint non-empty sets $K$ and $L$, and for each possible position of the $O_{j}$ 's in the $p_{j}$ 's relative to the members of $\mathcal{F}$, there are two sets $A_{x}$ and $A_{y}$ in $\mathcal{F}$ (that depend on the partition and on the position of the $O_{j}$ 's) so that for each $j \in K, l_{j}:\left(O_{j} * A_{x} * A_{y}\right)$, and for each $j \in L, l_{j}:\left(O_{j} * A_{y} * A_{x}\right)$-such a family of permutations is forbidden in $\mathbb{R}^{2 k-2}$.

\subsection{Construction of a Forbidden Family of Permutations}

We construct a family of $k$ permutations $\mathcal{P}=\left\{p_{1}, p_{2}, \ldots, p_{k}\right\}$ that has the property mentioned in Observation 5. The permutations involve $(k+1) \cdot\left(2^{k-1}+1\right)$ sets. In the first step we construct their subpermutations $\pi_{1}, \pi_{2}, \ldots, \pi_{k}$ which are permutations of $\left\{0,1, \ldots, 2^{k-1}\right\}$. 
Let $S_{1}, S_{2}, \ldots, S_{2^{k-1}}$ be the $2^{k-1}$ subsets of $\{1,2, \ldots, k\}$ that contain 1 (numbered in some way). Define $\pi_{1}, \pi_{2}, \ldots, \pi_{k}$ to be permutations of $\left\{0,1, \ldots, 2^{k-1}\right\}$ that satisfy:

In $\pi_{j}: \quad 0$ is before $i \Leftrightarrow j \in S_{i}$.

Note that the permutations $\pi_{1}, \pi_{2}, \ldots, \pi_{k}$ are not defined uniquely.

After that, for each $j \in\{1,2, \ldots, k\}$, construct a permutation $p_{j}$ by duplication of $\pi_{j} k+1$ times as follows: for $\pi_{j}=\left(\alpha_{0}, \alpha_{1}, \ldots, \alpha_{2^{k-1}}\right)$, define $p_{j}=\left(\left(\alpha_{0}, 1\right), \ldots\right.$, $\left.\left(\alpha_{2^{k-1}}, 1\right),\left(\alpha_{0}, 2\right), \ldots,\left(\alpha_{2^{k-1}}, 2\right), \ldots \ldots,\left(\alpha_{0}, k+1\right), \ldots,\left(\alpha_{2^{k-1}}, k+1\right)\right)$. The permutations $p_{1}, p_{2}, \ldots, p_{k}$ are permutations of the members of the set $\left\{0,1, \ldots, 2^{k-1}\right\} \times$ $\{1,2, \ldots, k+1\}$. For each $m \in\{1,2, \ldots, k+1\}$, we call the subpermutation $\left(\left(\alpha_{0}, m\right)\right.$, $\left.\ldots,\left(\alpha_{2^{k-1}}, m\right)\right)$ the $\mathrm{m}$ th interval of $p_{i}$.

Example of the Construction for $\boldsymbol{k}=3$. Let $S_{1}=\{1,2,3\}, S_{2}=\{1,2\}, S_{3}=\{1,3\}$, $S_{4}=\{1\}$. The permutations $\pi_{1}, \pi_{2}, \pi_{3}$ should be defined so that:

In $\pi_{1}: 0 \prec 1,0 \prec 2,0 \prec 3,0 \prec 4$.

In $\pi_{2}: 0 \prec 1,0 \prec 2,3 \prec 0,4 \prec 0$.

In $\pi_{3}: 0 \prec 1,2 \prec 0,0 \prec 3,4 \prec 0$.

For example, take $\pi_{1}=(01234), \pi_{2}=(34012), \pi_{3}=(24013)$.

Then, for these choices of $\pi_{1}, \pi_{2}$ and $\pi_{3}$,

$$
\begin{aligned}
p_{1}= & (\underbrace{(0,1),(1,1),(2,1),(3,1),(4,1)}_{1 \text { st interval }}, \underbrace{(0,2),(1,2),(2,2),(3,2),(4,2)}_{\text {2nd interval }}, \ldots, \\
& \ldots,(\underbrace{(0,4),(1,4),(2,4),(3,4),(4,4)),}_{4 \text { th interval }} \\
p_{2}= & ((3,1),(4,1),(0,1),(1,1),(2,1),(3,2),(4,2),(0,2),(1,2),(2,2), \ldots, \\
& \quad \ldots,(3,4),(4,4),(0,4),(1,4),(2,4)), \\
p_{3}= & ((2,1),(4,1),(0,1),(1,1),(3,1),(2,2),(4,2),(0,2),(1,2),(3,2), \ldots, \\
& \quad \ldots,(2,4),(4,4),(0,4),(1,4),(3,4)) .
\end{aligned}
$$

The family of permutations $\left\{p_{1}, p_{2}, p_{3}\right\}$ is forbidden in $\mathbb{R}^{4}$.

\subsection{Why the Construction Gives a Forbidden Family}

We prove that the family of permutations $\left\{p_{1}, p_{2}, \ldots, p_{k}\right\}$ defined in Section 3.5 is forbidden in $\mathbb{R}^{2 k-2}$. Suppose that there exists a family $\mathcal{F}$ of convex disjoint sets in $\mathbb{R}^{2 k-2}$ that admits $p_{1}, p_{2}, \ldots, p_{k}$ as geometric permutations. Let $l_{1}, l_{2}, \ldots, l_{k}$ be transversals giving these geometric permutations, and let $S$ be a $(k-2)$-flat that intersects each $l_{j}$, and let $O_{j} \in l_{j} \cap S$. Since each $p_{j}$ consists of $(k+1)$ "intervals", there is $m \in\{1,2, \ldots, k+1\}$ so that for each $j \in\{1,2, \ldots, k\}, O_{j}$ does not belong to the $m$ th interval of $p_{j}$. After dropping the "second component" $(m)$, the $m$ th interval of $p_{j}$ is identical to $\pi_{j}$, and $O_{j}$ is either before or after all of its sets. 
Let $K \cup L$ be a partition of $\{1,2, \ldots, k\}$ into two disjoint non-empty sets. Define $M=\left\{j \in\{1,2, \ldots, k\}: O_{j}\right.$ is before $\left.\pi_{j}\right\}$ and $N=\left\{j \in\{1,2, \ldots, k\}: O_{j}\right.$ is after $\left.\pi_{j}\right\}$. Define $K^{\prime}=(K \cap M) \cup(L \cap N)$. Note that $K^{\prime}$ is a subset of $\{1,2, \ldots, k\}$, and it is possible to assume that $1 \in K^{\prime}$ (otherwise we interchange $K$ and $L$ ). Hence $K^{\prime}=S_{a}$ for some $a \in\left\{1,2, \ldots, 2^{k-1}\right\}$.

Consider four cases:

- If $j \in K \cap M$, then $j \in K^{\prime}=S_{a}$, hence $l_{j}:\left(O_{j} \prec A_{0} \prec A_{a}\right)$.

- If $j \in K \cap N$, then $j \notin K^{\prime}=S_{a}$, hence $l_{j}:\left(A_{a} \prec A_{0} \prec O_{j}\right)$.

- If $j \in L \cap M$, then $j \notin K^{\prime}=S_{a}$, hence $l_{j}:\left(O_{j} \prec A_{a} \prec A_{0}\right)$.

- If $j \in L \cap N$, then $j \in K^{\prime}=S_{a}$, hence $l_{j}:\left(A_{0} \prec A_{a} \prec O_{j}\right)$.

In each case, for each $j \in K, l_{j}:\left(O_{j} * A_{0} * A_{a}\right)$, and for each $j \in L, l_{j}:\left(O_{j} * A_{a} * A_{0}\right)$. Then Observation 5 implies that the family of permutations is forbidden.

\section{Bounds on the Minimal Number of Sets in a Forbidden Family}

By Theorems 1 and 2, for each natural $d$, each family of $\lceil d / 2\rceil$ permutations is realizable in $\mathbb{R}^{d}$, but there is a forbidden family of $\lceil d / 2\rceil+1$ permutations. What is the minimal number of sets that must be involved in such a forbidden family? Denote this minimal number by $\varphi_{d}$. By our proof, $\varphi_{d} \leq(\lceil d / 2\rceil+2) \cdot\left(2^{\lceil d / 2\rceil}+1\right)$. This gives $\varphi_{2} \leq 9$ and $\varphi_{3}, \varphi_{4} \leq 20$, whereas, by the examples from Section $3.2, \varphi_{2} \leq 4, \varphi_{3} \leq 6$ and $\varphi_{4} \leq 9$.

On the other hand, for each natural $d$, there is a family of $d+1$ disjoint convex sets in $\mathbb{R}^{d}$ that have all possible $(d+1)$ !/2 geometric permutations [9]. It follows that $\varphi_{d} \geq d+2$. Thus, $\varphi_{2}=4,5 \leq \varphi_{3} \leq 6,6 \leq \varphi_{4} \leq 9$. It seems that the exponential upper bound for $\varphi_{d}$ can be improved substantially. It is clear that $d \leq d^{\prime}$ implies $\varphi_{d} \leq \varphi_{d^{\prime}}$. However, we do not even know whether $\varphi_{d}$ is strictly monotone as a function of $d$.

\section{References}

1. A. Asinowski, A. Holmsen, and M. Katchalski. The triples of geometric permutations for families of disjoint translations. Discrete Math. 241 (2001), 23-32.

2. A. Asinowski and M. Katchalski. The maximal number of geometric permutations for $n$ disjoint translates of a convex set in $\mathbb{R}^{3}$ is $\Omega(n)$. Submitted to Discrete Comput. Geom.

3. O. Cheong, X. Goaoc, and H.-S. Na. Geometric permutations of disjoint unit spheres. Accepted for publication in Comput. Geom. Theory Applic.

4. J. Eckhoff. Helly, Radon and Carathéodory type theorems. In Handbook of Convex Geometry, NorthHolland, Amsterdam, 1993, pp. 389-448.

5. H. Edelsbrunner and M. Sharir. The maximum number of ways to stab $n$ convex nonintersecting sets in the plane is $2 n-2$. Discrete Comput. Geom. 5 (1990), 35-42.

6. J.E. Goodman, R. Pollack, and R. Wenger. Geometric transversal theory. In New Trends in Discrete and Computational Geometry, J. Pach, ed., vol. 10 of Algorithms and Combinatorics, Springer-Verlag, Heidelberg, 1993, pp. 163-198.

7. A. Holmsen, M. Katchalski, and T. Lewis. A Helly-type theorem for line transversals to disjoint unit balls. Discrete Comput. Geom. 29 (2003), 595-602.

8. M. Katchalski, T. Lewis, and A. Liu. Geometric permutations of disjoint translates of convex sets. Discrete Math. 65 (1987), 249-260. 
9. M. Katchalski, T. Lewis, and A. Liu. The different ways of stabbing disjoint sets. Discrete Comput. Geom. 7 (1992), 197-206.

10. M. Katchalski, T. Lewis, and J. Zaks. Geometric permutations for convex sets. Discrete Math. 54 (1985), 271-284.

11. M. Katchalski, S. Suri, and Y. Zhou. A constant bound for geometric permutations of disjoint unit balls. Discrete Comput. Geom. 29 (2003), 161-173.

12. J. Radon. Mengen konvexer Körper, die einen gemeinsamen Punkt enthalten. Math. Ann. 83 (1921), 113-115.

13. S. Smorodinsky, J.S.B. Mitchell, and M. Sharir. Sharp bounds on geometric permutations of pairwise disjoint balls in $\mathbb{R}^{d}$. Discrete Comput. Geom. 23 (2000), 247-259.

14. H. Tverberg. Proof of Grünbaum's conjecture on common transversals for translates. Discrete Comput. Geom. 4 (1989), 191-203.

15. R. Wenger. Upper bounds on geometric permutations for convex sets. Discrete Comput. Geom. 5 (1990), 27-33.

16. R. Wenger. Helly-type theorems and geometric transversals. In Handbook of Discrete and Computational Geometry, CRC Press, Boca Raton, FL, 1997, pp. 63-82.

Received November 11, 2003, and in revised form March 3, 2004. Online publication July 26, 2004. 